\title{
A Comparison of Substance Use Behaviors of Hispanic Men by Sexual Orientation
}

\author{
Joseph P. De Santis ${ }^{1, *}$, Beatriz Valdes ${ }^{1,3}$, Elias Provencio-Vasquez ${ }^{2}$, Carol A. Patsdaughter ${ }^{3}$, \\ Karina A. Gattamorta ${ }^{1}$ \\ ${ }^{1}$ University of Miami School of Nursing \& Health Studies, Center of Excellence for Health Disparities Research: \\ EL CENTRO, Coral Gables, FL \\ ${ }^{2}$ University of Texas at El Paso School of Nursing, El Paso, TX \\ ${ }^{3}$ Florida International University College of Nursing \& Health Sciences, Miami, FL \\ *Corresponding Author: jdesantis@miami.edu
}

Copyright $(0) 2014$ Horizon Research Publishing All rights reserved.

\begin{abstract}
Background/Significance: Substance use is a public health concern in the United States. Hispanic men in the United States experience disproportionate rates of substance use when compared to other ethnic groups. Previous research with the general population of Hispanic men has identified factors that are related and may contribute to substance use. In addition, Hispanic men who have sex with men (HMSM) may experience additional social factors that may result in substance use. Despite the body of research on substance use among Hispanic men, no study to date has compared the substance use behaviors of Hispanic men by sexual orientation. Objectives: The purpose of this study was to compare the substance use behaviors of Hispanic men by sexual orientation.

Methods: A cross-sectional descriptive design was used to collect data from 164 community-dwelling Hispanic men (i.e., 77 heterosexual men and 87 HMSM) who resided in the South Florida area. Participants completed standardized measures of substance use and demographic characteristics. Results: Findings suggested that heterosexual men had higher rates of substance use when compared to MSM. No differences were found among the two groups of men in terms of alcohol intoxication. Religion, education, and income were not predictors of substance use. When health insurance status was controlled, MSM were less likely to report substance use. Implications: As a population, Hispanic men continue to experience health disparities in terms of substance use. Because substance use renders Hispanic men at risk for other health issues, more research is needed to understand the co-occurring health disparities experienced by Hispanic men who reside in the United States.
\end{abstract}

Keywords Hispanic Men, Sexual Orientation, Substance Use

\section{Introduction}

Substance use, which includes both the use of alcohol and drugs, is defined as the self-administration of a psychoactive substances that is that is not medically or legally sanctioned (World Health Organization [WHO], 1994). Substance use is a public health concern in the United States because the use of substances increases the risk of substance abuse. Currently about 22 million people in the United States are engaged in substance abuse. Substance abuse contributes to a host of psychosocial and legal issues and costs nearly $\$ 600$ billion annually (National Institute on Drug Abuse [NIDA], 2011).

Some evidence had suggested that Hispanics, the fastest growing ethnic group in the United States (Ortman \& Guarneri, 2009; U.S. Census Bureau, 2011), experience higher rates of alcohol and drug use when compared to other racial/ethnic groups (Gonzalez-Guarda et al., 2008; Substance Abuse and Mental Health Services Administration [SAMHSA], 2010; Zenmore, 2005). Among Hispanics residing in the United States, Hispanic men tend to have higher rates of substance use than Hispanic women (SAMHSA, 2010).

A few explanations for substance abuse among Hispanic men have been proposed. First, many Hispanics experience discrimination which results in stress. To cope with the stress of discrimination, some Hispanic men engage in substance use. Borrell and colleagues (2010) reported that experiences of discrimination increased the risk of alcohol use by $62 \%$ among Hispanic men. A second factor that may promote substance use among Hispanic is nativity. U.S.-born Hispanics have higher rates of substance use, substance abuse, and dependence on substances when compared to foreign-born Hispanics (SAMHSA, 2010). Closely linked to nativity is acculturation. Some studies have suggested that acculturation is associated with a lifetime risk of substance abuse disorders (Akins, Mosher, Smith, \& Gauthier, 2008; Ortega, Rosenheck, Alegria, \& Desai, 2000). Third, other factors such as religiosity and health insurance status may influence substance use. Religious networks are protective 
against tobacco, alcohol and marijuana use among Hispanic adolescents and young adults (Hodge et al., 2001; Hodge et al., 2011; Marsiglia Kulis, Niera, \& Patsai, 2006). Another study reported that Hispanics noted that health insurance, income, and education were predictors of treatment for alcohol abuse (Spence, Wallisch \& Smith, 2007).

Much like the general population of Hispanic men, substance use is a public health concern among Hispanic men who have sex with men (HMSM). A few studies have noted that high rates of substance use (De Santis, 2012; Fernandez, Jacobs, Warren, Sanchez, \& Bowen, 2009; Ramirez-Valles, Garcia, Campbell, Diaz, \& Heckathorn, 2008) and alcohol use (De Santis, 2012; De Santis, Arcia, Vermeesch, \& Gattamorta, 2011) among HMSM. In addition to discrimination experienced by the general population of Hispanic men (Borrell et al., 2006), HMSM may experience an additional layer of discrimination related to sexual orientation (Hightow-Weidman et al., 2011) that may contribute to substance use. In addition, other factors have been identified that attempt to explain why HMSM have high rates of substance use. These factors include acculturation, attachment to the gay community, a history of suicide attempts (Fernandez et al., 2007), loneliness, psychological distress, experiences of discrimination, and less social connections (Fernandez et al., 2009). Substance use among HMSM also has been linked to participation in high risk sex (De Santis, 2012; Fernandez et al., 2007; 2009).

\subsection{Substance Use and Sexual Orientation}

A few researchers have compared substance use by sexual orientation. Some studies reported that differences were noted in substance use when compared by sexual orientation. A large study that focused on a lifetime risk of psychiatric disorders (that included substance use/abuse) was conducted by Gilman and colleagues (2001). The researchers reported that same sex orientation was associated with substance use/abuse as well as anxiety, mood disorders, and suicidal thoughts and plans. A second study by Cochran and colleagues noted that sexual minorities have higher levels of substance abuse related to substance availability and tolerant social norms. Sexual orientation and substance abuse was mediated by perceptions of substance availability. Marshall and colleagues (2008) conducted a meta-analysis of research studies that focused on sexual orientation and substance use among adolescents. The researchers reported that sexual minorities have a 3-fold greater risk of substance use when compared to heterosexual adolescents. Of the 18 studies included in the meta-analysis, only one used a matched-comparison design. A third study sampled 34,653 American adults of both genders. This study reported that same sex sexual attraction is associated with an increased risk of substance use and dependence. Although the majority of those at risk for substance use and dependence did not meet diagnostic criteria for substance dependence, the risk was highest among gay women when compared to gay men, heterosexual men, and heterosexual women (McCabe et al.,
2009). A fourth study by Kecojevic and colleagues (2012) conducted a study that compared prescription drug misuse among high risk young adults in New York and Los Angeles. The researchers reported that sexual minorities were more likely to report opioid and tranquilizer misuse, but age at first use was the most significant predictor of drug misuse, regardless of sexual orientation.

Two research studies reported differences in substance use by sexual orientation and examined factors that were related to sexual orientation and that could influence substance use. These factors included cultural and environmental factors such as stigma and marginalization (Hughes, 2005). McCabe and colleagues (2010) reported that differences in substance use among gays and lesbians were related to discrimination that stemmed from sexual orientation, race, and gender.

Two studies noted that sexual orientation was not associated with an increased risk of substance abuse. One study reported that sexual experience among both heterosexuals and gays was the largest predictor of substance abuse (Brewster \& Harker-Tillman, 2012). A second larger study or 4,840 participants compared tobacco use, binge drinking, and marijuana use between heterosexual, gay, and bisexual college students of both genders. No differences were found in tobacco use, binge drinking, and marijuana use between heterosexual, gay, and bisexual males (Schauer, Berg \& Bryant 2013).

The available research on the relationship of substance use and sexual orientation remains unclear. In reviewing the existing literature, no studies are available that focus exclusively on men, but include women in the samples. Some of these studies did not report differences between males and females, but reported the results by sexual orientation without stratifying by gender. In addition, no studies could be located that compared substance use by sexual orientation among Hispanic men.

\section{Purpose}

Because no studies to date could be located that compared substance use behaviors among Hispanic men by sexual orientation, this study was designed to address this gap in the research literature. The following research questions for the study were developed:

1. How do substance use behaviors differ by sexual orientation among Hispanic men?

2. Do substance use behaviors among Hispanic men differ by sexual orientation when demographic factors such as income, education, and religion are controlled?

3. Do substance use behaviors among Hispanic men differ by sexual orientation when health insurance status is controlled?

4. Is there a difference in alcohol intoxication among Hispanic men when compared by sexual orientation?

\section{Method}




\subsection{Design}

This study used a descriptive, comparative design. This design was used to examine and compare characteristics of the two groups of Hispanic men using descriptive and inferential statistics. The study was approved by the Institutional Review Board for the Protection of Human Subjects at the University of Miami.

\subsection{Sample}

The sample included 164 community-dwelling Hispanic men in South Florida who participated in Project VIDA (i.e., Violence, Intimate Relationships, and Drugs Among Latinos), a large cross-sectional study that focused on interpersonal violence, sexual risk behaviors, and substance abuse among Hispanic men. The purpose of Project VIDA was to obtain baseline data on the aforementioned risk factors among a sample of both heterosexual Hispanic men and Hispanic MSM. This manuscript reports unpublished data on substance use behaviors.

Inclusion criteria for participation in Project VIDA included: (a) self-identification as a Hispanic or Latino man; (b) self-identification as heterosexual, gay/homosexual, or bisexual; (c) age 18 to 56; and (d) residence in South Florida for least a year before enrolling in the study.

The mean age of participants in this study was 41.25 years $(S D=9.03$, range 18-55). In terms of health insurance status, $38 \%(n=62 ; n=13$ heterosexual men vs. $n=49 \mathrm{MSM})$ reported current health insurance coverage. Additional demographic characteristics of the study sample are included in Table 1.

The sample $(N=164)$ was almost equally divided with respect to sexual orientation; 77 participants identified themselves as heterosexual, 63 participants identified themselves as gay/homosexual, 20 participants identified as bisexual, and 4 participants refused to disclose their sexual orientation. The four participants who refused to disclose their sexual orientation were excluded from data analysis, resulting in a final sample size of 164 participants: 77 heterosexual men (47\%) and 87 MSM (53\%).

For the purpose of this study, gay men, bisexual men, and those who refused to disclose their sexual orientation were grouped into the category of men who have sex with men (MSM). The inclusion of these men into the MSM category was based on a review of the last three sexual partners of these four participants, and it was found that all four participants reported at least one male sexual partner. Therefore, these four participants were included in the MSM group. This categorization of gay and bisexual men into the MSM category is consistent with the accepted definition of MSM: men who have any type of sexual behaviors with other males, regardless of self-identification. Some MSM prefer not to identify as gay or bisexual and may even self-identify as heterosexual despite sexual behaviors with other males. Hence, the term MSM focuses on sexual behaviors and not self-identification (Dowsett, 1990).

\subsection{Setting}

Data were collected from Hispanic men who resided in Miami-Dade County, Florida. This county's population is approximately $60 \%$ Hispanic, and at least $50 \%$ of the county's population is foreign-born (City Data, 2012). Multiple sites in this area were used to recruit participants including bars, barbershops, immigration offices, local stores, and street fairs.

Table 1. Demographic Characteristics of the Sample $(N=164)$

\begin{tabular}{|c|c|c|}
\hline Variable & Number & Percent \\
\hline \multicolumn{3}{|l|}{ Country of Origin } \\
\hline Cuba & 67 & 41 \\
\hline United States & 24 & 15 \\
\hline Puerto Rico & 23 & 14 \\
\hline Nicaragua & 13 & 8 \\
\hline Colombia & 10 & 6 \\
\hline Honduras & 10 & 6 \\
\hline Mexico & 7 & 4 \\
\hline Argentina & 2 & 1 \\
\hline Dominican Republic & 2 & 1 \\
\hline Peru & 2 & 1 \\
\hline Chile & 1 & 1 \\
\hline Costa Rica & 1 & 1 \\
\hline El Salvador & 1 & 1 \\
\hline \multirow[t]{2}{*}{ Ecuador } & 1 & 1 \\
\hline & Total No. (\%) & No. heterosexual and MSM \\
\hline \multicolumn{3}{|l|}{ Monthly Income ${ }^{a}$} \\
\hline$<\$ 999$ & $98(60 \%)$ & $\mathrm{n}=55 ; \mathrm{n}=43$ \\
\hline$\$ 1,000-\$ 3,000$ & $45(27 \%)$ & $\mathrm{n}=16 ; \mathrm{n}=29$ \\
\hline$>\$ 3,000$ & $3(8 \%)$ & $\mathrm{n}=2 ; \mathrm{n}=11$ \\
\hline \multicolumn{3}{|l|}{ Education } \\
\hline Less than high school & $46(28 \%)$ & $\mathrm{n}=28 ; \mathrm{n}=18$ \\
\hline High school & $43(26 \%)$ & $\mathrm{n}=28 ; \mathrm{n}=15$ \\
\hline College & $75(46 \%)$ & $\mathrm{n}=21 ; \mathrm{n}=54$ \\
\hline \multicolumn{3}{|l|}{ Religion } \\
\hline Catholic & $82(50 \%)$ & $n=36 ; n=46$ \\
\hline Christian & $41(25 \%)$ & $\mathrm{n}=24 ; \mathrm{n}=17$ \\
\hline Other & $14(8 \%)$ & $\mathrm{n}=7 ; \mathrm{n}=7$ \\
\hline None & $27(16 \%)$ & $\mathrm{n}=10 ; \mathrm{n}=17$ \\
\hline
\end{tabular}

Some data are missing: ${ }^{\mathrm{a}} n=156$

\subsection{Sampling}

The nonprobability sampling technique of convenience sampling was used. Once interviewed, participants were encouraged to refer other Hispanic men from their social networks, which allowed for the recruitment and enrollment of nearly equal numbers of Hispanic heterosexual men and 
Hispanic MSM.

\subsection{Instruments}

A demographic questionnaire and the Monitoring the Future (MTF) (Bachman, O’Malley \& Johnston, 1979) were used to collect the data in this study. The instrument and the demographic questionnaire were available in English and Spanish. Both were translated into Spanish, and then back-translated into English using the process described by Brislin (1970).

Substance use behaviors were measured using the MTF. The MTF has been used to collect data on substance use behaviors of high school students and adults to age 50 and has followed trends in substance abuse longitudinally since 1975. The MTF generates trends in substance use that are descriptive in nature, and exact psychometric properties of the MTF are not available, but the instrument's authors report over 30 years of data have been collected using the MTF (Bachman, Johnston, O’Malley, \& Schullenberg, 2006).

The MTF asks participants a set of questions about alcohol use and 14 other substances. Participants are asked three questions for each substance regarding the number of times substances were used during the lifetime, past 3 months, and during the last 30 days. For example, "On how many occasions (if any) have you used alcohol... (a) in your lifetime?, (b) past 3 months?, and (c) past 30 days? Participants are then provided with the following responses: $0,1-2,3-5,6-9,10-19,20-39$, and 40 or more.

The demographic questionnaire consisted of 21 questions regarding nativity, relationship status, living arrangements, number of children, sexual orientation, religion, educational level, employment status, income, health insurance status, perceived health status, and health provider information.

\subsection{Data Collection and Recording}

Potential participants were approached by a member of the research team and invited

to participate if they met inclusion criteria. Men who met inclusion criteria and who were interested in the study were provided with a business card that contained a telephone number that participants could call to schedule an appointment for data collection.
During the interview, the study was explained to the participants, and a signed informed consent was obtained in the participant's preferred language of English or Spanish. The interview was conducted in either English of Spanish based on the participant's preference. Because the participants were asked to provide sensitive data on substance use behaviors, the protection of privacy and confidentiality were important concerns. A Certificate of Confidentiality was obtained by the National Institutes of Health. Data were collected in a private office on campus or in a private area of the participant's home, depending on the participant's preference. A computerized data collection system was used primarily in an effort to decrease loss of data; however, some instances such as home visits required a manual collection on paper and data entry at a later time.

\section{Data Analysis}

The Statistical Package for the Social Sciences $\left(\mathrm{SPSS}^{\circledR}\right)$ Version 19.0 was utilized to analyze data (IBM SPSS Software, 2011). Substance use behaviors, a dichotomous variable coded as $0=$ no substance use during the lifetime and $1=$ substance use during the lifetime, was used as the dependent variable. The correlates of substance use were used as independent variables, and were tested in accordance to the research questions. The first research question involved testing for differences in substance use among Hispanic men by sexual orientation. The second research question controlled for the effects of income, education, and religion on sexual orientation and substance use. The third research question controlled for health insurance status and sexual orientation as related to substance use. The fourth question examined the number of occasions whereby participants consumed alcoholic beverages to the point of intoxication across the lifetime, past 3 months, and past 30 days by sexual orientation.

\section{Results}

Participants reported the use of substance during the lifetime, previous 3 months, and previous 30 days. The use of substance among the study's participants is detailed in Table 2. 
Table 2. Substance Use Rates among Hispanic Men $(N=164)$

\begin{tabular}{|c|c|c|c|}
\hline $\begin{array}{c}\text { Substance Type by sexual } \\
\text { orientation }\end{array}$ & $\begin{array}{c}\# \text { and } \% \text { reporting lifetime } \\
\text { use }\end{array}$ & $\begin{array}{c}\# \text { and } \% \text { reporting use in previous } 3 \\
\text { months }\end{array}$ & $\begin{array}{c}\# \text { and } \% \text { reporting use in the last } 30 \\
\text { days }\end{array}$ \\
\hline \multicolumn{4}{|l|}{ Marijuana } \\
\hline Heterosexual & $43(55.8)$ & $18(23.4)$ & $17(22.1)$ \\
\hline MSM & $30(34.5)$ & $10(11.5)$ & $10(11.5)$ \\
\hline \multicolumn{4}{|l|}{ LSD } \\
\hline Heterosexual & $11(14.3)$ & $11(14.3)$ & $0(0)$ \\
\hline MSM & $5(5.7)$ & $5(5.7)$ & $0(0)$ \\
\hline \multicolumn{4}{|l|}{ Psychedelics } \\
\hline Heterosexual & $9(11.7)$ & $9(11.7)$ & $0(0)$ \\
\hline MSM & $3(3.4)$ & $3(3.4)$ & $0(0)$ \\
\hline \multicolumn{4}{|l|}{ Crack Cocaine } \\
\hline Heterosexual & $26(33.8)$ & $12(15.6)$ & $11(14.3)$ \\
\hline MSM & $14(16.1)$ & $4(4.6)$ & $3(3.4)$ \\
\hline \multicolumn{4}{|l|}{ Cocaine } \\
\hline Heterosexual & $41(53.2)$ & $18(23.4)$ & $12(15.6)$ \\
\hline MSM & $22(25.3)$ & $3(3.4)$ & $2(2.3)$ \\
\hline \multicolumn{4}{|l|}{ "Uppers" } \\
\hline Heterosexual & $8(10.4)$ & $1(1.3)$ & $1(1.3)$ \\
\hline MSM & $7(8.0)$ & $1(1.1)$ & $1(1.1)$ \\
\hline \multicolumn{4}{|l|}{ Barbiturates } \\
\hline Heterosexual & $5(6.5)$ & $1(1.3)$ & $1(1.3)$ \\
\hline MSM & $6(6.9)$ & $2(2.3)$ & $2(2.3)$ \\
\hline \multicolumn{4}{|l|}{ Tranquilizers } \\
\hline Heterosexual & $15(19.5)$ & $9(11.7)$ & $9(11.7)$ \\
\hline MSM & $6(6.9)$ & $1(1.1)$ & $1(1.1)$ \\
\hline \multicolumn{4}{|l|}{ "Ecstasy" } \\
\hline Heterosexual & $10(13.0)$ & $0(0)$ & $0(0)$ \\
\hline MSM & $12(13.8)$ & $2(2.3)$ & $2(2.3)$ \\
\hline \multicolumn{4}{|l|}{ Methamphetamines } \\
\hline Heterosexual & $8(10.8)$ & $0(0)$ & $0(0)$ \\
\hline MSM & $8(9.2)$ & $1(1.1)$ & $1(1.1)$ \\
\hline \multicolumn{4}{|l|}{ Heroin } \\
\hline Heterosexual & $24(31.2)$ & $13(1.3)$ & $13(1.3)$ \\
\hline MSM & $4(4.6)$ & $0(0)$ & $0(0)$ \\
\hline \multicolumn{4}{|l|}{ Steroids } \\
\hline Heterosexual & $2(2.6)$ & $0(0)$ & $0(0)$ \\
\hline MSM & $4(4.6)$ & $2(2.3)$ & $2(2.3)$ \\
\hline \multicolumn{4}{|l|}{ Inhalants $^{\mathrm{a}}$} \\
\hline Heterosexual & $8(10.4)$ & $8(10.4)$ & $0(0)$ \\
\hline MSM & $3(3.4)$ & $3(3.4)$ & $0(0)$ \\
\hline \multicolumn{4}{|l|}{ Alcohol } \\
\hline Heterosexual & 49 (63.6) & $20(26.0)$ & $13(16.9)$ \\
\hline MSM & $57(65.5)$ & $22(25.3)$ & $15(17.2)$ \\
\hline
\end{tabular}

\footnotetext{
${ }^{\text {a }}$ Includes glue, aerosols, et cetera. Excludes cocaine.
} 
The first research question (How do substance abuse behaviors of Hispanic men differ by sexual orientation?) was tested using Chi-square analysis. In terms of sexual orientation, the demographic question asked the participants to chose from four categories to self-identify sexual orientation (i.e., heterosexual, homosexual, bisexual, and prefer not to answer). However, homosexual and bisexual men were collapsed into the category of MSM. Men who refused to answer $(n=4)$ were excluded from the analysis. A $2 \times 2$ contingency table of the data revealed a significant relationship between sexual orientation and substance use $\left[\chi^{2}\right.$ $(N=160,1)=7.38, p=.007]$, indicating that the heterosexual group reported more substance use than the MSM group.

The second research question (i.e., "Do substance use behaviors among Hispanic men differ by sexual orientation when demographic factors such as income, education, and religion are controlled?) were tested using binary logistic regression. The demographic questionnaire included the total monthly household income, including public assistance (i.e., after taxes). Participants were able to select from eight categories ranging from less than to $\$ 500$ to $\$ 6,000$ or more. Because total incomes were low, income was collapsed into two categories: less than or equal to $\$ 999(n=98)$ and $\$ 1,000$ or above $(n=58)$. For education, an open response was used for the years of education in the demographic intake form. Education was collapsed into two categories, high school or less and some college or more, assuming that high school education is equivalent to 12 years of education. Religion was collapsed from 10 categories to four predominant groupings, Catholic, Christian, other, and none. The dichotomized variables of income, education, and religion were entered as dummy coded variables in the first block of a hierarchical logistic regression analysis, with the dichotomized variable of sexual orientation entered in the second block. The demographic variables of religion, education, and income did not significantly predict substance use $-2 \mathrm{LL}=203.14, \chi^{2}(N=164,5)=6.92, p=.227$. The inclusion of sexual orientation was significant [-2LL = $\left.199.29, \chi^{2}(N=164,1)=3.85, p=.05\right]$. After controlling for income, education, and religion, MSM were approximately half as likely to report drug use $(O R=0.484, p=.051)$ than their heterosexual counterparts.

The third research question (Do substance use behaviors among Hispanic men differ by sexual orientation when health insurance status is controlled) was also tested using binary logistic regression. The demographic questionnaire queried whether or not participants had health insurance, and this variable was treated as a dichotomous variable. Health insurance status and sexual orientation combined to significantly predict drug use $\left[-2 \mathrm{LL}=212.67, \chi^{2}(N=164,2)\right.$ $=8.73, p=.013]$. After controlling for health insurance status, MSM were almost two thirds less likely to report drug use, $(O R=0.35, p=.004$ than their heterosexual counterparts.

Finally, the fourth research question (Is there a difference in alcohol intoxication among Hispanic men when compared by sexual orientation) was tested using Chi-square analysis.

Alcohol intoxication is defined as the use of alcoholic beverages which results in impaired physical and mental abilities. Alcohol intoxication was examined across the lifetime, past 3 months, and past 30 days. With regard to lifetime alcohol intoxication, no significant differences were found between the two groups of Hispanic men $\left[\chi^{2}(1, N=\right.$ $134)=1.79, p=.181]$. Additionally, rates of alcohol intoxication were examined for the past three months, $\left[\chi^{2}(1\right.$, $N=108), p=.947]$ and in the past 30 days $\left[\chi^{2}(1, N=52)=\right.$ $1.34, p=.246]$, and no significant differences were found by sexual orientation.

\section{Discussion}

This study compared substance use behaviors by sexual orientation in a sample of community-dwelling Hispanic men. The results of this study provide some important information on the substance abuse behaviors of Hispanic men. The majority of the participants in this study reported the use of various substances including alcohol. In fact, alcohol was the most common substance used by the study's participants, and $64.4 \%$ of the participants reported using alcohol at some point during the lifetime as compared to $51 \%$ reporting using a least one other substance in addition to alcohol during the lifetime. These findings support previous research that has reported that substance use is a major health issue among Hispanic men (SAMHSA, 2010). The rates of substance use in this sample of Hispanic men are difficult to interpret because no other data on substance use among other racial/ethnic groups was simultaneously collected that would allow comparisons of substance use between Hispanics men and men of other racial/ethnic groups. Therefore, it is not possible to know how the substance rates reported by the Hispanic men in this study compare to other groups of heterosexual men and MSM.

Because previous research is not available to document differences in substance use among Hispanic men by sexual orientation, the results of this study that noted that Hispanic MSM were less likely to engage in substance use when compared to heterosexual Hispanic men are again difficult to interpret. A number of previous studies have noted that Hispanic MSM engage in substance use (De Santis, 2012; Fernandez et al., 2009; Ramirez-Valles et al., 2008) and alcohol use (De Santis, 2012; De Santis et al., 2011), but it appears that substance use including alcohol use may not be higher in Hispanic MSM when compared to heterosexual Hispanic men in this sample. Of course, the sample size in this study was relatively small, so more research with larger samples is needed to continue the comparison of substance use behaviors among Hispanic men by sexual orientation.

If one examines the number of research studies that examined substance use behaviors of Hispanic MSM as compared to the studies that have focused on substance abuse among the general population of Hispanic men, more 
studies have focused on substance use among Hispanic MSM. This is not to suggest that Hispanic MSM have more psychopathology that may result in substance use, but an appreciation of the fact that stress from being in an ethnic minority combined with stress from being in a sexual minority may result in substance use to cope with this elevated stress (Shoptaw et al., 2009). Because a measure of stress was not included in this study, it is not possible to know how both types of minority stress might contribute to the substance use behaviors of Hispanic MSM. More research is needed to address this gap in the research knowledge base.

This study found that income, education, and religion could not be used to predict substance use behaviors among Hispanic men. Previous research noted that income, education, and religion were related to substance use among Hispanics (Hodge et al., 2001; Hodge et al., 2011; Marsiglia et al., 2006), but this was not supported by findings of this study. Given that religion is an important component of Hispanic culture (Levin, Markides \& Ray, 1996) and may be protective in terms of substance use and mental health (Hodge et al., 2001), this study's findings did not support these premises.

Health insurance status was found to be related to substance use among study participants. Previous research has found that health insurance was a predictor of substance abuse treatment (Spence et al., 2007). Because information was not collected to know if participants had received or were currently receiving substance abuse treatment, study findings on health insurance status cannot be directly compared to findings from previous research.

The findings on alcohol intoxication when compared by sexual orientation provide some new information on alcohol intoxication among Hispanic men. Study findings support previous research that has reported that gay men in relationships had no greater risk of heavy consumption of alcohol when compared to heterosexual men (Bloomfield et al., 2011). Although alcohol use may be a health risk for Hispanic MSM (De Santis et al., 2012), rates of alcohol use among Hispanic MSM may not be higher than rates for heterosexual Hispanic men. More research on alcohol intoxication among Hispanic men is warranted.

In terms of what is known about the relationship of substance use and sexual orientation among Hispanic men, the findings of this study provide some new information on how the substance use behaviors of Hispanic men may vary by sexual orientation. Previous research on substance use among Hispanic MSM has noted that these men engage in substance use

(De Santis, 2012; Fernandez et al., 2009; Ramirez-Valles et al., 2008) and alcohol use

(De Santis, 2012; De Santis et al., 2011). However, rates of substance use behaviors in this study were lower among Hispanic MSM when compared to Hispanic heterosexual men. Because literature is nonexistent that compares the substance use behaviors among these two groups of Hispanic men, it is difficult to make comparative interpretations of findings from this sample. Previous research with both Hispanic MSM and Hispanic heterosexual men has identified factors such as acculturation, depression, low self-esteem, and other mental health conditions that are related and may contribute to substance use and abuse (Gonzalez-Guarda, Florom-Smith, \& Thomas, 2011; Gonzalez-Guarda, Ortega, Vazquez, \& DeSantis, 2010). More research is needed to explore how these factors influence substance use when substance use behaviors are compared by sexual orientation among Hispanic men.

Although it was not the intent of this study to examine the influence of nativity on the substance use behaviors of Hispanic men, previous research on the influence of acculturation on substance use among Hispanic men has produced conflicting results (Provencio-Vasquez, Gonzalez-Guarda, \& De Santis, 2011). Because 76\% of study participants were foreign born, it is difficult to provide a meaningful comparison of how foreign birth and possible lower levels of acculturation might influence substance use behaviors of this sample. More research is needed to add to the knowledge base regarding the influence of acculturation on substance use behaviors of Hispanic men.

\section{Limitations}

There are a few limitations of this study. First, the descriptive comparative design was used to collect data at one time point and does not allow causal relationships to be explored with this data. Second, convenience sampling may have resulted in findings that cannot be generalized to the Hispanic male population of South Florida or to the Hispanic male population of the United States. Third, the sampling methodology used may have contributed to the homogeneous nature of the sample. A homogeneous sample could explain why relatively few differences were found between the two groups of Hispanic men. Fourth, the results could have been affected by the use of the MTF instrument. Despite the fact that the longitudinal data that have been collected with this instrument, psychometric properties of the MTF are not available, and did not allow comparison of this study's results to the original instrument.

A final limitation that requires acknowledgement is that information was only collected from participants on their sexual orientation. Data were not collected on sexual identification or sexual attraction. Previous research conducted with the general population of MSM has reported that incongruence often exists between orientation, self-identification, and behaviors. This incongruence may be one of the factors that contributes to the high rates of mental health issues (including substance use) that exists among MSM (Cochran et al. 2012; Marshall et al., 2008; McCabe et al., 2010). A more complete comparison of substance abuse behaviors between heterosexual Hispanic men and Hispanic MSM is necessary that includes sexual identification, sexual attraction, and sexual behaviors. Despite these limitations, 
this study provides some new findings that add to what is known about substance abuse among Hispanic men.

\section{Conclusions}

The results of this study provide some important clinical implications for nurses and other healthcare providers. Nurses and other clinicians need awareness that as a population, Hispanic men continue to experience health disparities in terms of substance use, which is a component of mental health.

The results of this study provide information for nurses and other healthcare providers to direct programs of health education geared to decrease health disparities related to substance use and abuse among Hispanic men, which will subsequently reduce morbidity and mortality of related to substance use. Because substance use and abuse render Hispanic men at risk for other health disparities, such as high risk sex and mental health conditions such as depression

(De Santis et al., 2011; Gonzalez-Guarda, et al., 2011; Provencio-Vasquez et al., 2011), nurses and other healthcare providers should screen Hispanic men not only for substance use problems, but also other related conditions that contribute to health disparities in this population of men.

\section{Acknowledgements}

This publication was made possible by Grant Number (1P60MD002255-01) from the National Center on Minority Health and Health Disparities of the National Institutes of Health. Its contents are solely the responsibility of the authors and do not necessarily represent the official views of the National Institutes of Health.

\section{REFERENCES}

[1] Akins, S., Mosher, C., Smith, C.L., \& Gauthier, J.F. (2008). The effect of acculturation on patterns of Hispanic substance use in Washington state. Journal of Drug Issues, 38(1), 103-118. doi:10.1177/002204260803800105

[2] Bachman, J. G., O'Malley, P. M., \& Johnston, L. D. (1979). Developing composite measures of drug use: Comparisons among lifetime, annual, and monthly prevalence reports for thirteen classes of drugs (Monitoring the Future Occasional Paper No. 5.). Ann Arbor, MI: Institute for Social Research.

[3] Bachman, J.G., Johnston, L.D., O'Malley, P.M., \& Schullenberg, J.E. (2006). The Monitoring the Future Project after 32 years: Design \& procedures. (Monitoring the Future Occasional Paper No. 64). Ann Arbor, MI: Institute for Social Research.

[4] Bloomfield, K., Wicki, M., Wilsnack, S., Hughes, T., \& Gmel, G. (2011). International differences in alcohol use according to sexual orientation. Substance Abuse, 32(4), 210- 219. doi:10.1080/08897077.2011.598404

[5] Brislin, R.W. (1970). Back-translation for cross-cultural research. Journal of Cross-Cultural Psychology, 1(3), 185-216. doi:10.11771135910457000100301.

[6] Borrell, L. N., Diez Roux, A.V., Jacobs, Jr., D. R., Shea, S., Jackson., S. A., Shrager, S., \& Blumental, R. S. (2010). Perceived racial/ethnic discrimination, smoking and alcohol consumption in the Multi-Ethnic Study of Atherosclerosis (MESA). Preventative Medicine, 51(3-4), 307-312. doi:10.1016/j.ypmed.2010.05.017

[7] Brewster, K.C., \& Harker-Tillman, K. (2012). Sexual orientation and substance use among adolescents and young adults. American Journal of Public Health, 102(6), 1168-1176.

[8] City Data. (2012). Miami-Dade county data. Retrieved from www.city-data.com/forum/miami/

[9] Cochran, S.D., Grella, C.E., \& Mays, V.M., (2012). Do substance use norms and perceived drug availability mediate sexual orientation differences in patterns of substance use? Results from the California Quality of Life Survey II. Journal of Studies in Alcohol and Drugs, 73(4), 675-685.

[10] De Santis, J. (2012). How do the sexual behaviors of foreign-born Hispanic men with have sex with men differ by relationship status? American Journal of Men's Health, 6(1), 6-17. doi:10.1177/1557988311403299

[11] De Santis, J. P., Arcia, A., Vermeesch, A., \& Gattamorta, K. A. (2011). Using structural equation modeling to identify predictors of sexual behaviors among Hispanic men who have sex with men. Nursing Clinics of North America, 46, 233-248. doi:10.1016/j.cnur.2011.02.010.

[12] Dowsett, G.W. (1990). Reaching men who have sex with men in Australia-an overview of AIDS education, community intervention and community attachment strategies. Australian Journal of Social Issues, 25, 186-195.

[13] Fernandez, M. I., Bowen, G. S., Warren, J.C., Ibanez, G. E., Hernandez, N., Harper, G. W., \& Prado, G. (2007). Crystal methamphetamine: A source of added sexual risk for Hispanic men who have sex with men. Drug \& Alcohol Dependence, $\quad 86, \quad 245-252$. doi:10.1016/j.drugalcdep.2006.06.016

[14] Fernandez, M. I., Jacobs, R. J., Warren, J. C., Sanchez, J., \& Bowen, G. S. (2009). Drug use and Hispanic men who have sex with men in South Florida: Implications for intervention development. AIDS Education \& Prevention, 21 (Suppl. 45), 45-60. doi:10.1521/aeap.2009.21.5.

[15] Gilman, S.E., Cochran, S.D., Mays, V.M., Hughes, M., Ostrow, D., \& Kessler, R.C. (2001). Risk of psychiatric disorders among individuals reporting same-sex partners in the National Comorbidity Study. American Journal of Public Health, 91(6), 933-999.

[16] Gonzalez-Guarda, R. M., Florom-Smith, A. L., \& Thomas, T. (2011). A syndemic model of substance abuse, intimate partner violence, HIV infection, and mental health among Hispanics. Public Health Nursing, 28(4), 366-378. doi:10.1111/j.1525-1445.2010.00928.x.

[17] Gonzalez-Guarda, R.M., Ortega, J., Vasquez, E.P., \& De Santis, J. (2010). La mancha negra: Substance abuse, violence and sexual risk among Hispanic males. Western 
Journal of Nursing Research, 32(1), 128-148. doi:10.1177/0193945909343594.

[18] Hodge, D. R., Cardenas, P., \& Montoya, H. (2001). Substance use: Spiritual and religious participation as protective factors among rural youth. Social Work Research, 25, 153-161. doi:10.1093/swr/25.3.153

[19] Hodge, D. R., Marsiglia, F. F., \& Nieri, T. (2011). Religion and substance use among youths of Mexican heritage: A social capital perspective. Social Work Research, 35(2), 137-146. doi:10.1093/swr/35.3.137

[20] Hughes, S.C. (2005). Alcohol use and alcohol-related problems among lesbians and gay men. Annual Review of Nursing Research, 23, 283-325.

[21] IBM SPSS Software. (2011). SPSS Base 19.0 for Windows user's guide [Computer Software]. Chicago, IL: Author.

[22] Kecojevic, A., Wong, C.F., Schranger, S.M., Silva, K., Bloom, J.J., Iverson, E., \& Lankenau, S.E. (2012). Initiation into prescription drug misuse: Differences between LGBT and heterosexual high risk young adults in Los Angeles and New York. Addictive Behaviors, 39(11), 1289-1293.

[23] Levin, J.S., Markides, K.S., \& Ray, L.A. (1996). Religious attendance and psychological well-being in Mexican-Americans: A panel analysis of three generations of data. Gerontologist, 36(4), 454-463. doi:10.1093/geront/36.4.454

[24] Marshall, M.P., Friedman, M.S., Stall, A., King, K.M., Miles, J., Gold, M.A. ... \& Morso, J.Q. (2008). Sexual orientation and adolescent substance use: A meta-analysis and methodological review. Addiction, 103(4), 546-563.

[25] McCabe, S.E., Bostwick, W.B., Hughes, T.L., West, B.T., \& Boyd, C.J. (2010). The relationship between discrimination and substance use disorders among lesbian, gay, and bisexual adults in the United States. American Journal of Public Health, 100(10), 1946-1952.

[26] McCabe, S.E., Hughes, T.L., Bostwick, W.B., West, B.T., \& Boyd, C.J. (2009). Sexual orientation, substance use behaviors and substance dependence in the United States. Addiction, 104(8), 1333-1345.

[27] Marsiglia, F.F., Kulis, S., Niera, T., \& Patsai, M. (2006). God forbid! Substance use among religious and non-religious youth. American Journal of Orthopsychiatry, 75, 585-598. doi:10.1037/0002-9432.75.4.585

[28] National Institute on Drug Abuse. (2011). NIDA infoFacts: Understanding drug abuse and addiction. Bethesda, MD: Author.

[29] Ortega, A.N., Rosenheck, R., Alegria, M., \& Desai, R.A. (2000). Acculturation and the lifetime risk of psychiatric and substance use disoders among Hispanics. Journal of Nervous \& Mental Disorders, 188(11), 728-735. doi:10.1097/00005053-200011000-00002

[30] Ortman, J. M., \& Guarneri, C. E. (2009). United States population projections: 2000 to 2050. Retrieved from http://www.census.gov/population/www/projections/analytic al-document09.pdf

[31] Provencio-Vasquez, E., Gonzalez-Guarda, R.M., \& De Santis, J.P. (2011). Acculturation, depression, self-esteem and substance abuse among Hispanic men. Issues in Mental Health Nursing, 32(2), 90-97. doi:10.3109/01612840.2010.528169.

[32] Ramirez-Valles, J., Garcia, D., Campbell, R. T., Diaz, R. M., \& Heckathorn, D. D. (2008). HIV infection, sexual risk behavior, and substance abuse among Latino gay and bisexual men and transgender persons. American Journal of Public Health, 98(6), 1036-1042. doi:10.2105/AJPH.2006.102624

[33] Schauer, G.L., Berg, C.J., \& Bryant, L.D. (2013). Sex differences in psychosocial correlates of concurrent substance use among substance use among heterosexual, homosexual, and bisexual college students. American Journal of Drug and Alcohol Abuse, (early edition), 1-7. DOI: 10.3109/00952990.2013.796962.

[34] Shoptaw, S., Weiss, R. E., Munjas, B., Hucks-Ortiz, C., Young, S. D. ... \& Gorbach, P. M. (2009). Homonegativity, substance use, sexual risk behaviors, and HIV status in poor and ethnic men who have sex with men in Los Angeles. Journal of Urban Health, 86(Supplement 1), 77-92. doi:10/1007/s11524-009-9372-5.

[35] Spence, R., Wallisch, L., \& Smith, S. (2007). Treatment seeking in populations in urban and rural settings on the border. Alcoholism: Clinical and Experimental Research, 31(6), 1002-1011. doi:10.1111/j.1530-0277.2007.00362.x

[36] Substance Abuse and Mental Health Services Administration (SAMHSA). (2010). The National Survey on Drug Use and Health (NSDUH) report: Substance abuse among Hispanic adults. Retrieved from http://www.oas.samhsa.gov/2k10/184/HispanicAdults.pdf

[37] World Health Organization (WHO). (1994). Lexicon of alcohol and drug terms. Geneva, Switzerland: Author.

[38] U.S. Census Bureau. (2011). 2010 census shows white population growth fueled by Hispanics. Retrieved from http://www.census.gov/newsroom/releases/archives/2010 ce nsus/cb11-cn184.html

[39] Zenmore, S.E. (2005). Re-examining whether and why acculturation related to drinking outcomes in a rigorous, national survey of Latinos. Alcoholism: Clinical and Experimental Research, 29(12), 2144-2153. doi:10.1097/01.alc.0000191775.01148.c0 